\title{
Hypersensitivity to Etoposide in Case of Metastatic Gestational Choriocarcinoma
}

\author{
Biljana Lazovića Vera Milenković $^{b}$ Marina Đelićc Sanja Mazićc \\ Katarina Jeremic ${ }^{b}$ Zlatko Hrgovićd \\ ${ }^{a}$ Department of Pulmonology, Clinical Hospital Center Zemun-Belgrade, ${ }^{b}$ Clinical Center \\ of Serbia, Institute of Gynecology and Obstetrics, University of Belgrade, Medical School, \\ and 'Institute of Physiology, Faculty of Medicine, University of Belgrade, Belgrade, Serbia; \\ ${ }^{d}$ Akademisches Lehrkrankenhaus der Johann-Wolfgang-Goethe-Universität Frankfurt am \\ Main, Frankfurt am Main, Germany
}

\section{Key Words}

Etoposide · Allergy · Gynecological malignancy

\begin{abstract}
Etoposide is commonly used in the treatment of a variety of neoplasms. Hypersensitivity reactions to etoposide are infrequently reported and include hypotension, hypertension, flushing, diaphoresis, chest discomfort, dyspnea, bronchospasm and loss of consciousness. We report the case of a 39-year-old woman who experienced acute bronchospasm, tachycardia, hypoxia and hypotension. The symptoms resolved within an hour after administration of intravenous fluids, methylprednisolone, diphenhydramine and oxygen. Subsequently, the patient was given etoposide phosphate without incident.
\end{abstract}

(c) 2013 S. Karger AG, Basel

\section{Introduction}

Etoposide is a cytotoxic agent (anticancer drug) which has been used in the treatment of a variety of malignant conditions. It is a derivative of podophyllin and acts by inhibiting mitosis. Etoposide is an essential component of chemotherapy in patients with rare gynecological malignancies such as germ cell tumors and gestational trophoblastic disease. It can be applied intravenously or orally in capsule form [1]. Etoposide is generally well tolerated. Common side effects include bone marrow suppression, alopecia and gastrointestinal symptoms [2]. Hypersensitivity reactions (HSR) to etoposide have been reported in 1- 
Lazović et al.: Hypersensitivity to Etoposide in Case of Metastatic Gestational Choriocarcinoma

3\% patients. In most patients, reactions arise within $10 \mathrm{~min}$ and are treated by discontinuing the infusion and administering antiallergic pharmacologic agents. The etiology of these reactions is still not known. They are believed to be of a nonimmunogenic origin [3]. There are no suitable substitutes for etoposide in regimens such as EMACO (etoposide, methotrexate, actinomycin D, cyclophosphamide, vincristine). Etoposide should only be discontinued when there is severe patient intolerance during a course of treatment, necessitating secondline therapy [4].

\section{Case Report}

A 39-year-old patient with a diagnosed recurrence of choriocarcionma in the site of vaginal scar tissue and distant metastases in the lungs and brain was admitted for treatment with a standard EMACO regimen. Two years earlier, she was diagnosed with choriocarcinoma with lung metastasis and was successfully treated with an EMACO regimen (8 cycles) and hysterectomy. The patient had no known allergies. This time, the council for trophoblastic disease prescribed 8 cycles of chemotherapy. On admission, she had severe anemia [red blood cells $3.89 \times 10^{12} / \mathrm{l}(4.10-5.10)$ and hemoglobin $\left.73.5 \mathrm{~g} / \mathrm{l}(115-165)\right]$, which required blood transfusion. During the second dose of blood transfusion, the patient felt heat and itching of the skin, with no other symptoms. The transfusion was stopped immediately. No additional therapy was administered. On the second day, the administration of etoposide was commenced $(200 \mathrm{mg}$ in $500 \mathrm{ml}$ of sodium chloride $0.9 \%$ over $60 \mathrm{~min}$ ). After 20 drops, the patient complained of generalized heat, tightness in the throat, shortness of breath and palpitations. She was found to be hypotensive (blood pressure $=100 / 60 \mathrm{~mm} \mathrm{Hg}$ ), tachycardic (pulse rate $=120$ beats $/ \mathrm{min}$ ) and desaturated to $84 \%$ on pulse oximetry. Physical examination showed bronchospasm of the bilateral lungs. Electrocardiography revealed only sinus tachycardia. In addition, the anesthesiologist noticed a discrete rush on the hand opposite to that used for transfusion. The infusion was ceased immediately. The patient was supplemented with oxygen and given fluids, methylprednisolone and diphenhydramine intravenously, which resolved her symptoms rapidly within $45 \mathrm{~min}$. The following day, the patient received premedication with methylprednisolone $(60 \mathrm{mg})$, ranitidine $(50 \mathrm{mg})$ and diphenhydramine $(50 \mathrm{mg}$ ) intravenously. Then, she was given an infusion with sodium chloride $0.9 \%$ over 10 min (placebo). The patient had no complaints. Next, an etoposide infusion was given. After a few drops of infusion, the patient developed acute dyspnea and chest tightness. The etoposide infusion was stopped immediately. Rescue medications were given as described previously. The symptoms resolved completely within $5 \mathrm{~min}$.

The following day the patient received an equivalent dose of etoposide phosphate intravenously, a water-soluble ester of etoposide, after the same premedication described above. She had no complaints and did not exhibit any further hypersensitivity symptoms. Further, the patient completed the total cycle of the EMACO regimen without complications.

\section{Discussion}

HSR to etoposide are uncommon, but can be life threatening. The etiology and the exact mechanism are unknown, but they are believed to be of nonimmunogenic origin. Nevertheless, there are some hypotheses that the concentration of the drug and the rate of infusion could explain HSR [5]. Another possible way of HSR is that the additive used in the preparation to dissolve etoposide (benzyl alcohol, polysorbate 80) may cause HSR. In animal models, 
polysorbate 80 has been shown to cause HSR by means of histamine release [6]. It is a type I reaction and antibody mediated. In our case, it is believed that the patient experienced the same type of reaction since the acute symptoms occurred immediately, as a result of releasing vasoactive substances such as histamine.

It was reported that the substitution of etoposide phosphate in patients with etoposide HSR seems to be appropriate. In the reported case, we have completed the treatment without any complications after changing to another pharmaceutical form of etoposide. Our patient was retreated using etoposide phosphate at an equivalent dose. The patient was premedicated, and no test dose of etoposide phosphate was administered. Etoposide phosphate is a water-soluble prodrug of etoposide, and this variant does not require polysorbate 80 to enter solution. To date, HSR to etoposide phosphate has not been reported $[3,7]$. In our case, it is likely that polysorbate 80 is a causative factor in the HSR to etoposide. However, another alternative to avoid any possibility of HSR is the use of oral instead of intravenous etoposide because this has a lower incidence of HSR. Also, a case of successful treatment of choriocarcinoma by diluting the etoposide concentration to half and reducing the drug administration rate by half to avoid HSR is described in the literature [8].

\section{Conclusion}

In situations where a patient expresses HSR to etoposide and where subsequent doses of etoposide are deemed necessary, replacement with etoposide phosphate is a reasonable and viable alternative with antihistamine and corticosteroid premedication.

\section{References}

$\checkmark 1$ Cortijo-Cascajares S, Jiménez-Cerezo MJ, Herreros de Tejada A: Review of hypersensitivity reactions to antineoplastic agents. Farm Hosp 2012;36:148-158.

2 Siderov J, Prasad P, De Boer R, Desai J: Safe administration of etoposide phosphate after hypersensitivity reaction to intravenous etoposide. Br J Cancer 2002;86:12-13.

3 Collier K, Schink C, Young AM, How K, Seckl M, Savage P: Successful treatment with etoposide phosphate in patients with previous etoposide hypersensitivity. J Oncol Pharm Pract 2008;14:51-55.

4 Siderov J, Prasad P, Boer DR, Desai J: Safe administration of etoposide phosphate after hypersensitivity reaction to intravenous etoposide. Br J Cancer 2002;86:12-13.

5 Kasperek C, Black CD: Two cases of suspected immunologic-based hypersensitivity reactions to etoposide therapy. Ann Pharmacother 1992;26:1227-1230.

-6 Coors EA, Seybold H, Merk HF, Mahler V: Polysorbate 80 in medical products and nonimmunologic anaphylactoid reactions. Ann Allergy Asthma Immunol 2005;95:593-599.

7 Aksahin A, Colak D, Altinbas M. Etoposide? Or polysorbate-80? Indian J Cancer 2011;48:272-273.

8 Taguchi A, Takeshita S, Machida R, Hori Y, Aida K, Furuya U, Ohe E, Murase T, Shinozuka N, Ayabe T, Mori H: Anaphylaxia induced by etoposide - a case report (in Japanese). Gan To Kagaku Ryoho 2003;30:1187-1189. 